\title{
Correction to: Paragangliomas arise through an autonomous vasculo-angio-neurogenic program inhibited by imatinib
}

\author{
Fabio Verginelli ${ }^{1,2} \cdot$ Silvia Perconti ${ }^{1,3} \cdot$ Simone Vespa $^{1,3} \cdot$ Francesca Schiavi $^{4} \cdot$ Sampath Chandra Prasad ${ }^{5}$. \\ Paola Lanuti ${ }^{1}$ - Alessandro Cama ${ }^{2} \cdot$ Lorenzo Tramontana $^{6}$. Diana Liberata Esposito ${ }^{1,3} \cdot$ Simone Guarnieri $^{1}$. \\ Artenca Sheu ${ }^{1,3} \cdot$ Mattia Russel Pantalone ${ }^{1,3} \cdot$ Rosalba Florio $^{2} \cdot$ Annalisa Morgano $^{1} \cdot$ Cosmo Rossi ${ }^{1}$. \\ Giuseppina Bologna ${ }^{1} \cdot$ Marco Marchisio ${ }^{1}$ - Andrea D'Argenio ${ }^{1} \cdot$ Elisa Taschin ${ }^{4} \cdot$ Rosa Visone $^{1,3} \cdot$ Giuseppe Opocher $^{4}$. \\ Angelo Veronese ${ }^{1,3} \cdot$ Carlo T. Paties $^{7} \cdot$ Vinagolu K. Rajasekhar $^{8} \cdot$ Cecilia Söderberg-Nauclér $^{9} \cdot$ Mario Sanna $^{5}$. \\ Lavinia Vittoria Lotti ${ }^{6} \cdot$ Renato Mariani-Costantini ${ }^{1,3}$ (D)
}

Published online: 1 February 2018

(c) Springer-Verlag GmbH Germany, part of Springer Nature 2018

\section{Correction to: Acta Neuropathologica}

https://doi.org/10.1007/s00401-017-1799-2

The given and family names of two co-authors were incorrect in the published article. The correct spelling should read as: Sampath Chandra Prasad and Vinagolu K. Rajasekhar.

The original article has been corrected.

The original article can be found online at https://doi.org/10.1007/ s00401-017-1799-2.

Renato Mariani-Costantini

rmc@unich.it

1 Laboratory of General Pathology, Center of Aging Science and Translational Medicine (CeSI-MeT), Gabriele d'Annunzio University, Via Luigi Polacchi 11, 66100 Chieti, Italy

2 Department of Pharmacy, Gabriele d'Annunzio University, Via dei Vestini 31, 66100 Chieti, Italy

3 Department of Medical, Oral and Biotechnological Sciences, Gabriele d'Annunzio University, Via dei Vestini 31, 66100 Chieti, Italy

4 Familial Cancer Clinic and Oncoendocrinology, Veneto Institute of Oncology, IRCCS, Padua, Italy

5 Otology and Skull Base Unit, Gruppo Otologico Piacenza-Roma, Via Antonio Emmanueli, 42, 29121 Piacenza, Italy
6 Department of Experimental Medicine, Sapienza University of Rome, Viale Regina Elena 324, 00161 Rome, Italy

7 Department of Oncology-Hematology, Service of Anatomic Pathology, Guglielmo da Saliceto Hospital, Via Taverna 49, 29100 Piacenza, Italy

8 Department of Surgery, Memorial Sloan Kettering Cancer Center, New York, NY 10065, USA

9 Department of Medicine, Experimental Cardiovascular Research Unit, and Department of Neurology, Center for Molecular Medicine, Karolinska Institute, Solnavägen 1, 17177 Solna, Stockholm, Sweden 